IJMMS 31:4 (2002) 215-227

PII. S0161171202109173

http://ijmms.hindawi.com

(c) Hindawi Publishing Corp.

\title{
$q$-HYPERELLIPTIC COMPACT NONORIENTABLE KLEIN SURFACES WITHOUT BOUNDARY
}

\author{
J. A. BUJALANCE and B. ESTRADA
}

Received 21 September 2001

\begin{abstract}
Let $X$ be a nonorientable Klein surface (KS in short), that is a compact nonorientable surface with a dianalytic structure defined on it. A Klein surface $X$ is said to be $q$-hyperelliptic if and only if there exists an involution $\Phi$ on $X$ (a dianalytic homeomorphism of order two) such that the quotient $X /\langle\Phi\rangle$ has algebraic genus $q$. $q$-hyperelliptic nonorientable KSs without boundary (nonorientable Riemann surfaces) were characterized by means of non-Euclidean crystallographic groups. In this paper, using that characterization, we determine bounds for the order of the automorphism group of a nonorientable $q$-hyperelliptic Klein surface $X$ such that $X /\langle\Phi\rangle$ has no boundary and prove that the bounds are attained. Besides, we obtain the dimension of the Teichmüller space associated to this type of surfaces.
\end{abstract}

2000 Mathematics Subject Classification: 30F50, $20 \mathrm{H} 10$.

1. Introduction. A Klein surface $X$ (KS in short) is a compact surface with a dianalytic structure defined on it [1]. Nonorientable KSs without boundary are also called nonorientable Riemann surfaces. A dianalytic homeomorphism of $X$ onto itself is called an automorphism of $X$. We say that a Klein surface $X$ is q-hyperelliptic if and only if there exists an involution $\Phi$ on $X$ such that the quotient $X /\langle\Phi\rangle$ has algebraic genus $q$. (If $q=0$, then $X$ is called hyperelliptic, and if $q=1$, then $X$ is elliptic-hyperelliptic.)

Let $X$ be a nonorientable $q$-hyperelliptic KS without boundary and $\operatorname{Aut}(x)$ the full group of its automorphisms. It is known [11] that $|\operatorname{Aut}(x)| \leq 84(p-1)$, where $p$ is the algebraic genus of $X$. This type of surfaces was characterized in [7] by means of non-Euclidean Crystallographic groups, and there, the bound 84( $p-1)$ was reduced to $12(p-1)$ if the quotient $X /\langle\Phi\rangle$ has boundary.

In this paper, we improve the bound $84(p-1)$ when $X /\langle\Phi\rangle$ has no boundary. The new bound will depend on the parity of $|\operatorname{Aut}(X)|$. That is made in Section 3. In Section 4 , we prove that the bounds are attained by showing different examples. In Section 5, the dimension of the Teichmüller space associated to this type of surfaces is obtained.

Similar characterizations for compact orientable KS without boundary (i.e., Riemann surfaces) and compact KS with boundary have been obtained in [2, 3, 4, 10].

2. Preliminaries. Denote by $\mathscr{D}$ the hyperbolic plane, and by $\mathscr{G}$ its group of isometries. A non-Euclidean crystallographic group $\Gamma$ (NEC group in short) is a discrete subgroup of $\mathscr{G}$ with compact quotient space $X=\mathscr{D} / \Gamma$. NEC groups were introduced by 
Wilkie [14] and Macbeath [9] associated to each NEC group a symbol, called signature, that determines its algebraic structure and has the following form:

$$
\sigma=\left(g ; \pm ;\left[m_{1}, \ldots, m_{r}\right] ;\left\{\left(n_{i 1}, \ldots, n_{i s_{i}}\right), i=1, \ldots, k\right\}\right),
$$

where $g, m_{i}$, and $n_{i j}$ are integers satisfying $g \geq 0, m_{i} \geq 2$, and $n_{i j} \geq 2 ; g$ is the topological genus of $X$. The sign determines the orientability of $X$. The numbers $m_{i}$ are the proper periods corresponding to cone points in $X$. The brackets $\left(n_{i 1}, \ldots, n_{i s_{i}}\right)$ are the period-cycles. The number $k$ of period-cycles is equal to the number of boundary components of $X$. The numbers $n_{i j}$ are the periods of the period-cycle $\left(n_{i 1}, \ldots, n_{i s_{i}}\right)$ also called link-periods, corresponding to corner points in the boundary of $X$. The number $p=\alpha g+k-1$ is called the algebraic genus of $X$, where $\alpha=1$ or 2 , if the sign of $\sigma(\Gamma)$ is "-" or "+", respectively. If the sign of $\sigma$ is "+" and $k=0$, then $\Gamma$ is a Fuchsian group.

An NEC group $\Gamma$ with signature $\sigma$ has the following presentation: Generators:

$$
\begin{array}{ll}
x_{i}, & i=1, \ldots, r ; \\
e_{i}, & i=1, \ldots, k ; \\
c_{i j}, & i=1, \ldots, k ; j=0, \ldots, s_{i} ; \\
a_{i}, b_{i}, & i=1, \ldots, g,(\text { if } \sigma \text { has sign }+ \text { ); } \\
d_{i}, & i=1, \ldots, g,(\text { if } \sigma \text { has sign }- \text { ). }
\end{array}
$$

Relations:

$$
\begin{gathered}
x_{i}^{m_{i}}, \quad i=1, \ldots, r ; \\
c_{i j-1}{ }^{2}=c_{i j}{ }^{2}=\left(c_{i j-1} c_{i j}\right)^{n_{i j}}, \quad i=1, \ldots, k ; j=1, \ldots, s_{i} ; \\
e_{i}^{-1} c_{i 0} e_{i} c_{i s_{i}}=1, \quad i=1, \ldots, k ; \\
x_{1} \cdots x_{r} e_{1} \cdots e_{k} a_{1} b_{1} a_{1}^{-1} b_{1}^{-1} \cdots a_{g} b_{g} a_{g}^{-1} b_{g}^{-1}=1 \quad(\text { if } \sigma \text { has sign }+), \\
x_{1} \cdots x_{r} e_{1} \cdots e_{k} d_{1}^{2} \cdots d_{g}^{2}=1 \quad(\text { if } \sigma \text { has sign }-) .
\end{gathered}
$$

It is known [9] that cyclic permutations of periods in period-cycles or arbitrary permutations of proper periods in the signature $\tau$ of an NEC group $\Gamma$ lead to a signature $\tau^{\prime}$ of an NEC group $\Gamma^{\prime}$ isomorphic to $\Gamma$. Every $\Gamma$ with signature (2.1) has associated a fundamental region whose area, $|\Gamma|$, is also called the area of the group [12]:

$$
|\Gamma|=2 \pi\left(\alpha g+k-2+\sum_{i=1}^{r}\left(1-\frac{1}{m_{i}}\right)+\frac{1}{2} \sum_{i=1}^{k} \sum_{j=1}^{s_{i}}\left(1-\frac{1}{n_{i j}}\right)\right) .
$$

An NEC group $\Gamma$ with signature $\sigma$ actually exists if and only if the right-hand side of (2.4) is greater than zero [15]. If $\Gamma^{\prime}$ is a subgroup of an NEC group $\Gamma$ of finite index $N$, then it is also an NEC group and the following Hurwitz-Riemann formula holds:

$$
\left|\Gamma^{\prime}\right|=N|\Gamma|
$$


Let $X$ be a nonorientable Klein surface without boundary of topological genus $g \geq 3$. Then by [11], there exists an NEC group $\Gamma$ with signature

$$
(g ;-;[-] ;\{-\})
$$

such that $X=\mathscr{D} / \Gamma$.

An NEC group with signature (2.6) is said to be a nonorientable surface group. In [11] it was proved that if $G$ is a group of automorphisms of a nonorientable Klein surface without boundary $X=\mathscr{D} / \Gamma$, then $G$ can be presented as a quotient $\Gamma^{\prime} / \Gamma$, for some NEC group $\Gamma^{\prime}$ such that $\Gamma \triangleleft \Gamma^{\prime}$. The full group of automorphisms of $X$ is $\operatorname{Aut}(X)=N_{\mathscr{c}}(\Gamma) / \Gamma$, where $N_{\mathscr{G}}(\Gamma)$ is the normalizer of $\Gamma$ in $\varphi$.

In general, it is very difficult to decide whether a group of automorphisms $G=\Gamma^{\prime} / \Gamma$ of a Klein surface $X=\mathscr{D} / \Gamma$ equals $\operatorname{Aut}(X)$ or not. This problem is equivalent to the problem of deciding whether $\Gamma^{\prime}$ equals the normalizer of $\Gamma$ in $\mathscr{G}$ or not.

From now on, KS is assumed to have algebraic genus not smaller than 2.

The following results, that appear in [7], will be used through this paper.

THEOREM 2.1. Let $X=D / \Gamma$ be a nonorientable $K S$ without boundary of algebraic genus $p$. Then, $X$ is q-hyperelliptic if and only if there exists an NEC group $\Gamma_{1}$, containing $\Gamma$ as a subgroup of index 2 and having the signature of one of the following types:

(a) $\left(h ;+;\left[2,{ }^{(p+1)-2 q}, 2\right] ;\left\{(-)^{q-2 h+1}\right\}\right), 0 \leq h \leq q / 2$;

(b) $\left(h ;-;[2,(p+1)-2 q, 2] ;\left\{(-)^{q-h+1}\right\}\right), 0 \leq h \leq q$ if $p$ is even, $1 \leq h \leq q+1$ if $p$ is odd. Moreover,

(1) for each $0 \leq h \leq q / 2$, there exists a nonorientable q-hyperelliptic KS without boundary $D / \Gamma$ whose $q$-hyperellipticity group has the signature (a);

(2) for each $0 \leq h \leq q$ (if $p$ is even) and for each $1 \leq h \leq q+1$ (if $p$ is odd), there exists a nonorientable q-hyperelliptic KS without boundary $D / \Gamma$ whose $q$ hyperellipticity group has the signature (b).

THEOREM 2.2. Let $X=D / \Gamma$ be a nonorientable q-hyperelliptic KS without boundary of algebraic genus $p>4 q+1$, then the $q$-hyperelliptic involution $\Phi$ is central and unique in $\operatorname{Aut}(X)$.

3. Bounds for the order of the automorphism group. Let $X=\mathscr{D} / \Gamma$ be a nonorientable $q$-hyperelliptic KS without boundary, and let $\Phi$ be the $q$-hyperellipticity involution. Then $\langle\Phi\rangle \simeq \Gamma_{1} / \Gamma$ for a certain NEC group $\Gamma_{1}$ and, by Theorem $2.1, X /\langle\Phi\rangle \simeq \mathscr{D} / \Gamma_{1}$ is an orientable KS with boundary, or a nonorientable KS (with or without boundary).

A group of automorphisms of $X$ can be represented as a quotient $\Gamma^{\prime} / \Gamma$ where $\Gamma \triangleleft \Gamma^{\prime}$. Since $\Phi$ is central and unique, we have $\Gamma \triangleleft \Gamma_{1} \triangleleft \Gamma^{\prime}$.

In the next theorem we prove that the bound $84(g-1)$, for the order of an automorphism group of a nonorientable KS without boundary $X$, can be improved when $X$ is $q$-hyperelliptic and $X /\langle\Phi\rangle$ has empty boundary.

THEOREM 3.1. Let $X=D / \Gamma$ be a nonorientable q-hyperelliptic $K S$ without boundary of algebraic genus $p>4 q+1$. Let $\Phi$ be the $q$-hyperelliptic involution and $X /\langle\Phi\rangle a$ surface without boundary. Then,

(1) if $|\operatorname{Aut}(X)|=2 N, N$ is odd, then $|\operatorname{Aut}(X)| \leq 6(q-1)$, 
(2) if $|\operatorname{Aut}(X)|=2 N, N$ is even, and

(a) signature of $\Gamma^{\prime}$ has sign "-", then $|\operatorname{Aut}(X)| \leq 12(q-1)$,

(b) signature of $\Gamma^{\prime}$ has sign " + ", then $|\operatorname{Aut}(X)| \leq 24(q-1)$.

Proof. Since $\sigma(\Gamma)=(p+1 ;-;[-]\{-\}), p>4 q+1$, and $X /\langle\Phi\rangle$ has no boundary, by Theorem 2.1 there exists a unique NEC group $\Gamma_{1}$ with the signature

$$
\sigma\left(\Gamma_{1}\right)=(q+1 ;-;[2,(p+1)-2 q, 2]), \quad p \text { is odd }
$$

(I) Suppose $\left[\Gamma^{\prime}: \Gamma_{1}\right]=N$ is odd. We know by [5, Chapter 2] that the signature of $\Gamma^{\prime}$ is

$$
\sigma\left(\Gamma^{\prime}\right)=\left(g^{\prime} ;-;\left[m_{1}, \ldots, m_{t}\right]\right)
$$

Let $x_{i} \in \Gamma^{\prime}, i=1, \ldots, t$, be the generators of proper-periods such that $p_{i}$ is the smallest integer satisfying $x_{i}^{p} \in \Gamma_{1}\left(p_{i} \neq 0\right)$. As the only elements of finite order in $\Gamma_{1}$ have order two, then (see [5, Section 2.2])

$$
p_{i}=\frac{m_{i}}{2} \quad \text { or } \quad p_{i}=m_{i}
$$

We can suppose, without loss of generality, that for $1 \leq i \leq n, p_{i}=m_{i} / 2$ and for $n+1 \leq i \leq t, p_{i}=m_{i}$. Thus, by [5, Section 2.2]

$$
p-2 q+1=\sum_{i=1}^{n}\left(\frac{N}{p_{i}}\right)=\sum_{i=1}^{n}\left(\frac{2 N}{m_{i}}\right) .
$$

From the Riemann-Hurwitz formula $\left|\Gamma_{1}\right|=N\left|\Gamma^{\prime}\right|$, we have

$$
(q-1)+\frac{(p-2 q+1)}{2}=N\left[\left(g^{\prime}-2\right)+\sum_{i=1}^{n}\left(1-\frac{1}{m_{i}}\right)+\sum_{i=n+1}^{t}\left(1-\frac{1}{m_{i}}\right)\right] .
$$

By (3.4) and (3.5),

$$
\begin{aligned}
(q-1) & =N\left[\left(g^{\prime}-2\right)+\sum_{i=1}^{n}\left(1-\frac{1}{m_{i}}\right)-\sum_{i=1}^{n}\left(\frac{1}{m_{i}}\right)+\sum_{i=1}^{t}\left(1-\frac{1}{m_{i}}\right)\right] \\
& =N\left[\left(g^{\prime}-2\right)+\sum_{i=1}^{n}\left(1-\frac{2}{m_{i}}\right)+\sum_{i=n+1}^{t}\left(1-\frac{1}{m_{i}}\right)\right],
\end{aligned}
$$

therefore,

$$
N=\frac{\left|\Gamma_{1}\right|}{\left|\Gamma^{\prime}\right|}=\frac{(q-1)}{\left(g^{\prime}-2\right)+\sum_{i=1}^{n}\left(1-2 / m_{i}\right)+\sum_{i=n+1}^{t}\left(1-1 / m_{i}\right)} .
$$


Since $p>4 q+1$, then by (3.4) we have

$$
\sum_{i=1}^{n}\left(\frac{N}{m_{i}}\right)>(q+1)
$$

We fix $q$ in (3.7) and denote

$$
A=g^{\prime}-2+\sum_{i=1}^{n}\left(1-\frac{2}{m_{i}}\right)+\sum_{i=n+1}^{t}\left(1-\frac{1}{m_{i}}\right), \quad 2 \leq m_{i} \leq \infty .
$$

We will consider several cases to determine the minimum positive value of $A$ (that makes $N$ maximal). For $n$ and $t$ fix

(i) if $g^{\prime} \geq 2$, then $A^{(1)} \geq \sum_{i=1}^{n}\left(1-2 / m_{i}\right)+\sum_{i=n+1}^{t}\left(1-1 / m_{i}\right)>0$;

(ii) if $g^{\prime}=1$, then

$$
\begin{aligned}
A^{(2)} & =\sum_{i=1}^{n}\left(1-\frac{2}{m_{i}}\right)+\sum_{i=n+1}^{t}\left(1-\frac{1}{m_{i}}\right)-1>0, \\
A^{(1)} & >A^{(2)}>0 \\
A^{(2)} & \geq k_{2}\left(1-\frac{2}{2}\right)+k_{4}\left(1-\frac{2}{4}\right)+k_{6}\left(1-\frac{2}{6}\right)+k_{2}^{\prime}\left(1-\frac{1}{2}\right)+k_{3}^{\prime}\left(1-\frac{1}{3}\right)-1 \\
& =\frac{1}{2}\left(k_{4}+k_{2}^{\prime}\right)+\frac{2}{3}\left(k_{6}+k_{3}^{\prime}\right)-1 ;
\end{aligned}
$$

where $k_{4}, k_{6}, k_{2}^{\prime}$, and $k_{3}^{\prime}$ are nonnegative integers with $k_{4}+k_{6}+k_{2}^{\prime}+k_{3}^{\prime} \leq t$. The values of $k_{i}$ and $k_{i}^{\prime}$, for which the above expression is minimum occurs only for $k_{4}+k_{2}^{\prime}=1$, $k_{6}+k_{3}^{\prime}=1$, but these imply that $N$ is even, a contradiction. Then, the suitable ones are $k_{4}+k_{2}^{\prime}=0$ and $k_{6}+k_{3}^{\prime}=2$, that is,

$$
\begin{array}{ll}
k_{6}=2, & k_{4}=k_{2}^{\prime}=k_{3}^{\prime}=0 ; \\
k_{3}^{\prime}=2, & k_{4}=k_{6}=k_{2}^{\prime}=0 ; \\
k_{3}^{\prime}=k_{6}=1, & k_{2}^{\prime}=k_{4}=0 ;
\end{array}
$$

and $N_{(\max )}=3(q-1)$. Consequently, $|\operatorname{Aut}(X)| \leq 6(q-1)$ and the signature of $\Gamma^{\prime}$ has one of the following signatures:

$$
\begin{array}{lll}
\boldsymbol{T}_{1}=\left(1,-,\left[2, \ldots, k_{2}, 2,6,6\right]\right), & q>\frac{4}{3} \frac{1}{k_{2}}+1, & p=\left(3 k_{2}+4\right)(q-1)+1 ; \\
\boldsymbol{T}_{2}=(1,-,[2, \ldots, \ldots, 2,3,3]), & q>\left(\frac{3 k_{2}+2}{3 k_{2}-2}\right), & p=(q-1)\left(3 k_{2}+2\right)+1 ; \\
\boldsymbol{T}_{3}=(1,-,[2, \ldots, 2,2,6,3]), & q>\left(\frac{3 k_{2}+3}{3 k_{2}-1}\right), & p=(q-1)\left(3 k_{2}+3\right)+1 .
\end{array}
$$

(II) Now, suppose that $\left[\Gamma^{\prime}: \Gamma_{1}\right]=N$ is even. We know (see $[5,6]$ ) that the signature of $\Gamma^{\prime}$ is

$$
\sigma\left(\Gamma^{\prime}\right)=\left(g^{\prime} ; \pm ;\left[m_{1}, \ldots, m_{t}\right] ;\left\{\left(n_{i 1}, \ldots, n_{i s_{i}}\right), i=1, \ldots, k\right\}\right)
$$


The proper-periods of $\Gamma_{1}$ may proceed from elliptic elements $x_{i}$ or period-cycles of $\Gamma^{\prime}$. If $p_{i}$ is the order of $\Gamma_{1} x_{i} \in\left(\Gamma^{\prime} / \Gamma_{1}\right)$, then $x_{i}$ generates in $\Gamma_{1}, \sum_{1}^{n}\left(N / p_{i}\right)$ proper periods, all of them being equal to 2 .

Now consider the periods in $\Gamma_{1}$ provided by reflections of $\Gamma^{\prime}$ not in $\Gamma_{1}$. Let $q_{i j}$ be the smallest integer for which $\left(c_{i j}, c_{i j-1}\right)^{q_{i j} \in \Gamma_{1}}$, since the only elements of finite order are those of order 2 , we have $\left(n_{i j} / q_{i j}\right)=2$ or 1 . Let $E=\left\{(i, j) \mid\left(n_{i j} / q_{i j}\right)=2\right\}$. Clearly, the only proper-periods in $\Gamma_{1}$ induced by reflections of $\Gamma^{\prime}$ are those provided by pairs of reflections corresponding to elements of $E$, and each such pair produces $\left(N / 2 q_{i j}\right)$ periods, all of them being equal to 2 . As a result, we obtain $\sum_{(i, j) \in E}\left(N / 2 q_{i j}\right)$ properperiods in $\Gamma_{1}$. Then by (3.4)

$$
p-2 q+1=\sum_{1}^{n}\left(\frac{2 N}{m_{i}}\right)+\sum_{(i, j) \in E}\left(\frac{N}{2 q_{i j}}\right) .
$$

(A) If the sign in the signature of $\Gamma^{\prime}$ is minus, by the Riemann-Hurwitz formula, we hold the expression

$$
\begin{aligned}
N^{(-)} & =\frac{\left|\Gamma_{1}\right|}{\left|\Gamma^{\prime}\right|} \\
& =\frac{(q-1)}{g^{\prime}+k-2+\sum_{i=1}^{n}\left(1-\frac{2}{m_{i}}\right)+\sum_{n+1}^{t}\left(1-\frac{1}{m_{i}}\right)+\frac{1}{2} \sum_{(i, j) \in E}\left(1-\frac{2}{n_{i j}}\right)+\frac{1}{2} \sum_{(i, j) \notin E}\left(1-\frac{1}{n_{i j}}\right)} .
\end{aligned}
$$

Since $p>4 q+1$, then by (3.14) we have

$$
\sum_{1}^{n}\left(\frac{N^{(-)}}{m_{i}}\right)+\sum_{(i, j) \in E}\left(\frac{N^{(-)}}{2 n_{i j}}\right)>(q+1) .
$$

As in previous case ( $N$ odd), we are looking for the value of parameters in (3.15), that makes $N^{(-)}$the maximum possible. Let

$$
A=g^{\prime}-2+B_{1}+B_{2},
$$

where

$$
\begin{aligned}
& B_{1}=\sum_{i=1}^{n}\left(1-\frac{2}{m_{i}}\right)+\sum_{i=n+1}^{t}\left(1-\frac{1}{m_{i}}\right), \\
& B_{2}=k+\frac{1}{2} \sum_{(i, j) \in E}\left(1-\frac{2}{n_{i j}}\right)+\frac{1}{2} \sum_{(i, j) \notin E}\left(1-\frac{1}{n_{i j}}\right) .
\end{aligned}
$$

For $n, t, k$, and $E$ fix, we have the following cases:

(i) if $g^{\prime} \geq 2: A^{(1)} \geq B_{1}+B_{2}$;

(ii) if $g^{\prime}=1$ : $A^{(2)}=B_{1}+B_{2}-1$. (Obviously $A^{(1)} \geq A^{(2)}$.)

As we have seen, in the case where $N$ is odd,

$$
B_{1} \geq B_{1}^{*}=\frac{1}{2}\left(k_{4}+k_{2}{ }^{\prime}\right)+\frac{2}{3}\left(k_{6}+k_{3}{ }^{\prime}\right)+0 k_{2} .
$$


The minimum positive value $A^{*}$ of $A$ occurs when $k=0, k_{4}+k_{2}^{\prime}=1$, and $k_{6}+k_{3}^{\prime}=1$ in $B_{1}^{*}$; so $B_{1}^{*}=7 / 6$ and $A^{*}=7 / 6-1=1 / 6 \leq B_{1}(\min )-1 \leq B_{1}(\min )+B_{2}(\min )-1$.

Therefore, $N(\max )=6(q-1)$ and

$$
|\operatorname{Aut}(X)| \leq 12(q-1)
$$

The possible values of $k_{4}, k_{2}^{\prime}, k_{6}$, and $k_{3}^{\prime}$, lead us to conclude that $\Gamma^{\prime}$ has one of the following signatures:

$$
\begin{array}{lll}
\tau_{4}=\left(1,-,\left[2, k_{2}, 2,4,6\right]\right), & q>\left(\frac{6 k_{2}+9}{6 k_{2}+5}\right), & p=\left(6 k_{2}+7\right)(q-1)+1 ; \\
\tau_{5}=\left(1,-,\left[2, k_{2}, 2,2,6\right]\right), & q>\left(\frac{6 k_{2}+2}{2 k_{2}}\right), & p=\left(6 k_{2}+4\right)(q-1)+1 ; \\
\tau_{6}=\left(1,-,\left[2, k_{2}, 2,4,3\right]\right), & q>\left(\frac{6 k_{2}+5}{6 k_{2}+1}\right), & p=\left(6 k_{2}+5\right)(q-1)+1 ; \\
\tau_{7}=\left(1,-,\left[2, k_{2}, 2,2,3\right]\right), & q>\left(\frac{3 k_{2}+1}{3 k_{2}-1}\right), & p=\left(6 k_{2}+2\right)(q-1)+1 .
\end{array}
$$

(B) If the sign in the signature of $\Gamma^{\prime}$ is positive, we have, by the Riemann-Hurwitz formula, the following expression:

$$
N^{(+)}=\frac{\left|\Gamma_{1}\right|}{|\Gamma|}=\frac{(q-1)}{2 g^{\prime}-2+B_{1}+B_{2}},
$$

where $B_{1}$ and $B_{2}$ are as in (3.18).

Let $A=2 g^{\prime}-2+B_{1}+B_{2}$. As in the previous case we are going to minimize $A$. We have for $n, t, k$, and $E$ fix the following cases.

CASE 1. If $g \geq 1$, then $A \geq B_{1}+B_{2}$.

CASE 2. If $g=0$, then $A \geq A^{*}=B_{1}+B_{2}-2$.

Since the sign in the signature of $\Gamma_{1}$ is negative, then $\Gamma^{\prime}$ must have some period-cycle, that is, $k \neq 0$.

If $t=0$, then $A^{*}=B_{2}-2$ and

$$
\begin{aligned}
B_{2} & =1+\frac{1}{2} \sum_{(i, j) \in E}\left(1-\frac{2}{n_{i j}}\right)+\frac{1}{2} \sum_{(i, j) \notin E}\left(1-\frac{1}{n_{i j}}\right) \\
& \geq 1+\frac{1}{2} N_{2}\left(1-\frac{2}{2}\right)+\frac{1}{2} N_{4}\left(1-\frac{2}{4}\right)+\frac{1}{2} N_{6}\left(1-\frac{2}{6}\right)+\frac{1}{2} N_{2}^{\prime}\left(1-\frac{1}{2}\right)+\frac{1}{2} N_{3}^{\prime}\left(1-\frac{1}{3}\right) \\
& =1+\frac{1}{4}\left(N_{4}+N_{2}^{\prime}\right)+\frac{1}{3}\left(N_{6}+N_{3}^{\prime}\right),
\end{aligned}
$$


then

$$
A^{*} \geq \frac{1}{4}\left(N_{4}+N_{2}^{\prime}\right)+\frac{1}{3}\left(N_{6}+N_{3}^{\prime}\right)+0 N_{2}-1>0
$$

where the nonnegative integers $N_{i}$ are the number of $n_{i j}=i,(i, j) \in E$, and $N_{i}^{\prime}$ is the number of $n_{i j}=i,(i, j) \notin E$. The value of (3.24) will be minimum for

$$
N_{4}+N_{2}^{\prime}=3, \quad N_{6}+N_{3}^{\prime}=1,
$$

so, $N(\max )=12(q-1)$ and

$$
|\operatorname{Aut}(X)| \leq 24(q-1)
$$

From conditions (3.25), we obtain the following signatures for $\Gamma^{\prime}$ :

$$
\begin{aligned}
& \tau_{8}=\left(0,+,[-],\left\{\left(2, N_{2}, 2,2,4,4,6\right)\right\}\right), \\
& \boldsymbol{T}_{9}=\left(0,+,[-],\left\{\left(2, N_{2}, 2,2,4,4,3\right)\right\}\right), \\
& \tau_{10}=\left(0,+,[-],\left\{\left(2, \ldots N_{2}, 2,2,2,4,6\right)\right\}\right) \text {, } \\
& \boldsymbol{T}_{11}=\left(0,+,[-],\left\{\left(2, N_{2}, 2,2,2,4,3\right)\right\}\right), \\
& \boldsymbol{T}_{12}=(0,+,[-],\{(2, \ldots, \ldots, 2,2,2,2,6)\}) \text {, } \\
& \boldsymbol{T}_{13}=(0,+,[-],\{(2, \ldots, 2,2,2,2,3)\}), \\
& \boldsymbol{T}_{14}=\left(0,+,[-],\left\{\left(2, N_{2}, 2,4,4,4,6\right)\right\}\right) \text {, } \\
& \boldsymbol{T}_{15}=\left(0,+,[-],\left\{\left(2, N_{2}, 2,4,4,4,3\right)\right\}\right) \text {. }
\end{aligned}
$$

Now, if $t \neq 0$, then $A^{*}=B_{1}+B_{2}-2$ and

$$
A^{*}=\frac{1}{2}\left(k_{4}+k_{2}^{\prime}\right)+\frac{2}{3}\left(k_{6}+k_{3}^{\prime}\right)+\frac{1}{4}\left(N_{4}+N_{2}^{\prime}\right)+\frac{1}{3}\left(N_{6}+N_{3}^{\prime}\right)-1>0 .
$$

The smallest value of $A$ appears under the following conditions:

$$
k_{6}+k_{3}^{\prime}=0, \quad N_{6}+N_{3}^{\prime}=1, \quad N_{4}+N_{2}^{\prime}=3, \quad k_{4}+k_{2}^{\prime}=0
$$

or

$$
k_{6}+k_{3}^{\prime}=0, \quad N_{6}+N_{3}^{\prime}=1, \quad N_{4}+N_{2}^{\prime}=1, \quad k_{4}+k_{2}^{\prime}=1 .
$$

Then $N_{(\max )}=12(q-1)$ and

$$
|\operatorname{Aut}(X)| \leq 24(q-1)
$$


By elementary combinatorial methods, we obtain 16 solutions for the signature of $\Gamma^{\prime}$

$$
\begin{aligned}
& \tau_{16}=\left(0,+,\left[2, k_{2}, 2\right],\left\{\left(2, N_{2}, 2,2,2,2,6\right)\right\}\right), \\
& \boldsymbol{T}_{17}=\left(0,+,\left[2, \ldots k_{2}, 2\right],\{(2, \ldots, 2,2,2,4,6)\}\right), \\
& \boldsymbol{T}_{18}=\left(0,+,\left[2, k_{2}, 2\right],\left\{\left(2, \ldots, N_{2}, 2,2,4,4,6\right)\right\}\right), \\
& \boldsymbol{T}_{19}=\left(0,+,\left[2, \stackrel{k_{2}}{\ldots}, 2\right],\left\{\left(2, N_{2}, 2,4,4,4,6\right)\right\}\right), \\
& \boldsymbol{T}_{20}=\left(0,+,\left[2, \stackrel{k_{2}}{.}, 2,2\right],\left\{\left(2, \stackrel{N_{2}}{.}, 2,2,6\right)\right\}\right), \\
& \boldsymbol{T}_{21}=\left(0,+,\left[2, k_{2}, 2,2\right],\left\{\left(2, N_{2}, 2,4,6\right)\right\}\right), \\
& \boldsymbol{T}_{22}=\left(0,+,\left[2, \stackrel{k_{2}}{.}, 2,4\right],\left\{\left(2, N_{2}, 2,2,6\right)\right\}\right), \\
& \boldsymbol{T}_{23}=\left(0,+,\left[2, \stackrel{k_{2}}{.}, 2,4\right],\left\{\left(2, \ldots N_{2}, 2,4,6\right)\right\}\right), \\
& \boldsymbol{T}_{24}=\left(0,+,\left[2, k_{2}, 2\right],\left\{\left(2, N_{2}, 2,2,2,2,3\right)\right\}\right), \\
& \boldsymbol{T}_{25}=\left(0,+,\left[2, \stackrel{k_{2}}{\cdots}, 2\right],\left\{\left(2, \stackrel{N_{2}}{.}, 2,2,2,4,3\right)\right\}\right), \\
& \boldsymbol{T}_{26}=\left(0,+,\left[2, k_{2}, 2\right],\left\{\left(2, N_{2}, 2,2,4,4,3\right)\right\}\right), \\
& \boldsymbol{T}_{27}=\left(0,+,\left[2, k_{2}, 2\right],\left\{\left(2, N_{2}, 2,4,4,4,3\right)\right\}\right), \\
& \boldsymbol{T}_{28}=(0,+,[2, \ldots, \ldots, 2,2],\{(2, \ldots, \ldots, 2,2,3)\}), \\
& \boldsymbol{T}_{29}=\left(0,+,\left[2, \ldots k_{2}, 2,2\right],\left\{\left(2, N_{2}, 2,4,3\right)\right\}\right), \\
& \boldsymbol{T}_{30}=\left(0,+,[2, \ldots, \ldots, 2,4],\left\{\left(2, \ldots, N_{2}, 2,2,3\right)\right\}\right), \\
& \boldsymbol{T}_{31}=\left(0,+,\left[2, \stackrel{k_{2}}{.}, 2,4\right],\left\{\left(2, N_{2}, 2,4,3\right)\right\}\right) \text {. }
\end{aligned}
$$

COROLLARY 3.2. Let $X=D / \Gamma$ be a nonorientable $q$-hyperelliptic KS without boundary of algebraic genus $p>4 q+1$ and $|\operatorname{Aut}(X)|=\Gamma^{\prime} / \Gamma$. Let $\Phi$ be the q-hyperelliptic involution such that $X /\langle\Phi\rangle$ has no boundary. Then,

(1) if $|\operatorname{Aut}(X)|=2 N, N$ is odd, then $|\operatorname{Aut}(X)| \leq(3 / 2)(p-6)$;

(2) if $|\operatorname{Aut}(X)|=2 N, N$ is even, and

(a) signature of $\Gamma^{\prime}$ has sign " " ", then $|\operatorname{Aut}(X)| \leq 3(p-6)$,

(b) signature of $\Gamma^{\prime}$ has sign "+", then $|\operatorname{Aut}(X)| \leq 6(p-6)$.

4. Examples. It is natural to ask for what values of $q$ the bounds of Theorem 3.1 are attained. We have found examples for low values of $q$. Here we present some examples of surfaces with maximal automorphism group for the three different bounds.

In the remainder of this section, $\theta$ denotes the canonical epimorphism from $\Gamma^{\prime}$ onto $G \simeq \Gamma^{\prime} / \Gamma$, where $\Gamma^{\prime}$ has one of the signatures obtained in Section $3 \tau_{1}, \ldots, \tau_{31}$. We denote by $\Phi$ the $q$-hyperelliptic involution, $\pi: G \rightarrow G /\langle\Phi\rangle$ the natural epimorphism and $\bar{\theta}=\pi \theta$.

The procedure we are going to follow in these examples is as follows:

(a) to prove the existence of epimorphisms $\theta$ and $\bar{\theta}$ as above, such that $\operatorname{ker} \bar{\theta} \simeq \Gamma_{1}$, $\operatorname{ker}(\theta) \simeq \Gamma$, and $\bar{\theta}=\pi \theta$;

(b) consequently, we obtain the realization (fulfillment) of the automorphism group $G \simeq \Gamma^{\prime} / \Gamma$ which attains the bound. 
EXAMPLE 4.1. If $N$ is odd, $\sigma\left(\Gamma^{\prime}\right)=(1,-,[2,2,6,6])$, and $q=2$, then $|\operatorname{Aut}(X)| \leq 6$. Let $\bar{\theta}: \Gamma^{\prime} \rightarrow \bar{G} \simeq \Gamma^{\prime} / \Gamma_{1}$ be the canonical epimorphism defined as follows:

$$
\bar{\theta}\left(d_{1}\right)=1, \quad \bar{\theta}\left(x_{1}\right)=\bar{y}, \quad \bar{\theta}\left(x_{2}\right)=\bar{y}^{-1}, \quad \bar{\theta}\left(x_{3}\right)=\bar{\theta}\left(x_{4}\right)=\overline{1},
$$

where $d_{1}, x_{1}, x_{2}, x_{3}$, and $x_{4}$ are the generators of $\Gamma^{\prime}$, and $\bar{y}$ is an element of order three in $\Gamma^{\prime} / \Gamma_{1}$. As $\left|\Gamma^{\prime} / \Gamma_{1}\right|=3$ and it is a group generated by $\bar{y}$, with the relation $\bar{y}^{3}=1$, we have $\Gamma^{\prime} / \Gamma_{1} \simeq C_{3}$; so that $G \simeq\left(\Gamma^{\prime} / \Gamma\right)$ would have order 6 and its quotient by a subgroup generated by a central element must be $C_{3}$. Then $\Gamma^{\prime} / \Gamma$ is $C_{6}$.

Finally, we need to prove that there exists a nonorientable 2-hyperelliptic KS without boundary of topological genus $p=11$ whose automorphism group is $C_{6}$.

Let $\theta$ be the epimorphism $\theta: \Gamma^{\prime} \rightarrow C_{6} \simeq\left\langle y: y^{6}=1\right\rangle$ defined by

$$
\theta\left(d_{1}\right)=1, \quad \theta\left(x_{1}\right)=y, \quad \theta\left(x_{2}\right)=y^{-1}, \quad \theta\left(x_{3}\right)=\theta\left(x_{4}\right)=y^{3} .
$$

We choose $\Phi=y^{3}$ as the central element, thus $\operatorname{ker} \theta \simeq \Gamma$. Moreover, it is straightforward to prove $\operatorname{ker} \bar{\theta} \simeq \theta^{-1}\left(\left\langle y^{3}\right\rangle\right) \simeq \Gamma_{1}$.

EXAMPLE 4.2. Let $N$ be even, $\sigma\left(\Gamma^{\prime}\right)=(1,-,[2,2,2,3])$, and $q=3$. Let $\theta$ and $\bar{\theta}$ be the canonical epimorphisms over

$$
\begin{aligned}
G & \simeq\left\langle x, y, z: x^{2}, y^{3}, z^{2},(x y)^{3},[x z],[y z]\right\rangle \simeq A_{4} \times C_{2}, \\
\bar{G} & \simeq\left\langle x, y: x^{2}, y^{3},(x y)^{3}\right\rangle \simeq A_{4},
\end{aligned}
$$

respectively,

$$
\begin{aligned}
& \theta\left(d_{1}\right)=x y, \quad \theta\left(x_{1}\right)=\Phi, \quad \theta\left(x_{2}\right)=\Phi, \quad \theta\left(x_{3}\right)=x, \quad \theta\left(x_{4}\right)=y ; \\
& \bar{\theta}\left(d_{1}\right)=\bar{x} \bar{y}, \quad \bar{\theta}\left(x_{1}\right)=\overline{1}, \quad \bar{\theta}\left(x_{2}\right)=\overline{1}, \quad \bar{\theta}\left(x_{3}\right)=\bar{x}, \quad \bar{\theta}\left(x_{4}\right)=\bar{y} .
\end{aligned}
$$

It is easy to see that $\operatorname{Ker}(\bar{\theta}) \simeq(4,-,[2,2,2,2],\{-\})$ and $X=\mathscr{D} / \operatorname{Ker}(\theta)$ is a nonorientable surface without boundary of algebraic genus $p=29$. Now, consider $\Phi=z$. Since $z$ is central in $G$ we have $G /\langle\Phi\rangle=\bar{G}$ and $X /\langle\Phi\rangle$ has algebraic genus 3, therefore, $X$ is a 3-hyperelliptic Klein surface such that $|\operatorname{Aut}(X)|=12(q-1)=24$.

EXAMPLE 4.3. Let $N$ be even, $\sigma\left(\Gamma^{\prime}\right)=(0,+,[2],\{(2,2,2,6)\})$, and $q=2$. Let $G, \theta, \bar{G}$, and $\bar{\theta}$ be defined as follows:

$$
\begin{gathered}
G=\left\langle x, y, z: x^{2}, y^{2}, z^{2},(x y)^{2},(y z)^{2},(z x)^{6}\right\rangle \simeq D_{6} \times C_{2} ; \\
\theta\left(x_{1}\right)=\theta\left(e_{1}\right)=\Phi, \quad \theta\left(c_{1,0}\right)=\theta\left(c_{1,4}\right)=x, \quad \theta\left(c_{1,1}\right)=\Phi x y, \\
\theta\left(c_{1,2}\right)=y, \quad \theta\left(c_{1,3}\right)=z ; \\
\bar{G}=\left\langle\bar{x}, \bar{y}, \bar{z}: \bar{x}^{2}, \bar{y}^{2}, \bar{z}^{2},(\bar{x} \bar{y})^{2},(\bar{y} \bar{z})^{2},(\bar{z} \bar{x})^{3}\right\rangle \simeq D_{3} \times C_{2}, \\
\bar{\theta}\left(x_{1}\right)=\bar{\theta}\left(e_{1}\right)=\overline{1}, \quad \bar{\theta}\left(c_{1,0}\right)=\bar{\theta}\left(c_{1,4}\right)=\bar{x}, \quad \bar{\theta}\left(c_{1,1}\right)=\bar{x} \bar{y}, \\
\bar{\theta}\left(c_{1,2}\right)=\bar{y}, \quad \bar{\theta}\left(c_{1,2}\right)=\bar{z} .
\end{gathered}
$$


We can see that $\operatorname{Ker}(\bar{\theta})$ has signature $\tau=(3,-,[2, \ldots, 2],\{-\})$ and $X=\mathscr{D} / \operatorname{Ker}(\theta)$ is a nonorientable surface without boundary and algebraic genus $p=17$. Consider $\Phi=$ $(z x)^{3}$ an order-two central element in $G$, we have $G / \Phi=\bar{G}$ and $X\langle\Phi\rangle$ has signature $\tau$. In particular, $X /\langle\Phi\rangle$ has algebraic genus 2, then by Theorem $2.1 X$ is a 2-hyperelliptic Klein surface. Furthermore, $G$ is an automorphism group of $X$. Now, by Theorem 3.1, we have $\operatorname{Aut}(X) \leq 24(q-1)$, then $G=\operatorname{Aut}(X)$ attaining the upper bound.

It remains as an open problem to determine topological types of nonorientable $q$-hyperelliptic Klein surfaces without boundary admitting maximal automorphism group for arbitrary $q$.

5. Teichmüller space. In this section, we are going to study the dimension of the Teichmüller space associated to nonorientable $q$-hyperelliptic Klein surfaces without boundary of algebraic genus $p>4 q+1$ such that $X /\langle\Phi\rangle$ has empty boundary. We denote this space by $T_{q}^{-p}$.

Let $\Gamma$ be an NEC group. We define the Weil space associated to $\Gamma, R(\Gamma)$, as the set of monomorphisms $r: \Gamma \rightarrow \mathscr{G}$ such that $r(\Gamma)$ is discrete and $\mathscr{D} / r(\Gamma)$ is compact.

We say that two elements $r_{1}, r_{2} \in R(\Gamma)$ are equivalents if there exists $g \in \varphi$ such that $r_{1}(\Gamma)=g r_{2}(\Gamma) g^{-1}$. The quotient space by this relation is called the Teichmüller space of $\Gamma$ and denoted by $T(\Gamma)$. When $\Gamma$ is a Fuchsian group (only orientation preserving elements) with signature $\left(g,+,\left[m_{1}, \ldots, m_{r}\right]\right)$, it is known that $T(\Gamma)$ is a cell of dimension $d(\Gamma)=6(g-1)+2 r$. Singerman [13] proved that if $\Gamma$ is a proper NEC group, then $d(\Gamma)=(1 / 2) d\left(\Gamma^{+}\right)$where $\Gamma^{+}$is the canonical Fuchsian group associated to $\Gamma$. The Teichmüller Modular group of $\Gamma, M(\Gamma)$, is the quotient group $\operatorname{Aut}(\Gamma) / I(\Gamma)$ where $\operatorname{Aut}(\Gamma)$ is the full group of automorphisms of $\Gamma$ and $I(\Gamma)$ the set of inner automorphisms. The Modular group $M(\Gamma)$ acts on $T(\Gamma)$ as follows: if $[\tau] \in T(\Gamma)$ and $\bar{\alpha} \in M(\Gamma)$, then $\bar{\alpha}[\tau]=[\tau \cdot \alpha]$.

Let $X=\mathscr{D} / \Gamma$ be a nonorientable $q$-hyperelliptic Klein surfaces without boundary of algebraic genus $p>4 q+1$ such that $X /\langle\Phi\rangle$ has empty boundary. Following Harvey [8],

$$
T_{q}^{-p}=\bigcup_{\bar{\alpha} \in M(\Gamma)} \bar{\alpha}\left(\bigcup_{\Phi \in \Phi\left(\Gamma, \Gamma_{1}, \Gamma_{1} / \Gamma\right)} \operatorname{Im} \hat{\imath}_{\phi}\right),
$$

where $\Gamma_{1}$ is the group of the $q$-hyperellipticity of $X$ with signature

$$
\left(q+1,-,\left[2,{ }^{p+1-2 q}, 2\right],\{-\}\right), \quad \Phi\left(\Gamma, \Gamma_{1}, \Gamma_{1} / \Gamma\right)
$$

is the set of all equivalence classes of surjections $\phi: \Gamma_{1} \rightarrow \mathbb{Z}_{2}$ with $\operatorname{Ker}(\phi)=\Gamma$ modulo the action of $\operatorname{Aut}\left(\Gamma_{1}\right)$ and $\operatorname{Aut}\left(\mathbb{Z}_{2}\right)$, and $\operatorname{Im} \hat{\imath}_{\phi}$ is the image set $\hat{\imath}_{\phi}\left(T\left(\Gamma_{1}\right)\right)$ under the isometry $\hat{\imath}_{\phi}$ induced by the inclusion $i_{\phi}: \operatorname{Ker} \phi \rightarrow \Gamma_{1}$.

Here the family $\Phi\left(\Gamma, \Gamma_{1}, \Gamma_{1} / \Gamma\right)$ consists of $q+2$ classes each of them characterized by the number of glide reflections between the canonical generators of $\Gamma_{1}$ in $\operatorname{Ker} \phi$ (to know $d_{1}, \ldots, d_{q+1}$ ). If we call these classes $\left[\phi_{i}\right], i=0, \ldots, q+1$; a representative of each 
class is defined by

$$
\begin{array}{ll}
\phi_{i}\left(d_{j}\right)=\overline{0} & \text { for } j=1, \ldots, i \\
\phi_{i}\left(d_{j}\right)=\overline{1} & \text { for } j=i+1, \ldots, q+1 \\
\phi_{i}\left(x_{j}\right)=\overline{1} & \text { for } j=1, \ldots, p+1-2 q .
\end{array}
$$

Then,

$$
T_{q}^{-p}=\bigcup_{\bar{\alpha} \in M(\Gamma)} \bar{\alpha}\left(\operatorname{Im} \hat{\imath}_{0} \cup \cdots \cup \operatorname{Im} \hat{\imath}_{q+1}\right)
$$

where $\hat{\imath}_{j}: T\left(\Gamma_{1}\right) \hookrightarrow T(\Gamma), \tau \in R\left(\Gamma_{1}\right)[\tau] \longmapsto\left[\tau \cdot i_{j}\right]$ is the real analytic homeomorphism (see [8]) induced by the inclusion $i_{j}: \operatorname{Ker} \phi_{j} \rightarrow \Gamma_{1}$.

The situation allows us to use Maclachlan's method (see [10, Lemma 3]), to prove that (5.4) is a disjoint union.

Suppose that there exist $l, m \in\{0, \ldots, q+1\}$, such that

$$
\bar{\alpha} \hat{\imath}_{l}\left(T\left(\Gamma_{1}\right)\right) \cap \hat{\imath}_{m}\left(T\left(\Gamma_{1}\right)\right) \neq \varnothing .
$$

Therefore, there exist $\tau_{1}, \tau_{2} \in R\left(\Gamma_{1}\right)$ satisfying

$$
\left[\tau_{1} \cdot i_{l} \cdot \alpha\right]=\left[\tau_{2} \cdot i_{m}\right]
$$

if and only if there exists $t \in \mathscr{G}$ such that

$$
\tau_{1} \cdot i_{l} \cdot \alpha(f)=t\left(\tau_{2} \cdot i_{m}\right)(f) t^{-1}, \quad \forall f \in \Gamma_{1} .
$$

Consider $\tau_{3}: \tau_{3}(f)=t \tau_{2}(f) t^{-1}$, for all $f \in \Gamma_{1}$; then $\tau_{1} \cdot i_{l} \cdot \alpha=\tau_{3} \cdot i_{m}$. Now, as $\Gamma_{1}$ is unique we can consider the inverse image of $\tau_{3}$ to obtain

$$
\left(\tau_{3}{ }^{-1} \cdot \tau_{1}\right) \cdot i_{l} \cdot \alpha=i_{m}
$$

being $\tau_{3}{ }^{-1} \cdot \tau_{1}$ an automorphism of $\Gamma_{1}$. This leads us to conclude that $l=m$. Thus, $T_{q}^{-p}$ is a disjoint union of copies $\hat{\imath}_{l}\left(T\left(\Gamma_{1}\right)\right)$, that is, $T_{q}^{-p}$ is a submanifold of $T(\Gamma)$ of dimension

$$
d\left(\Gamma_{1}\right)=2 p-q-1
$$

ACKNOWLEDGment. The authors wish to thank Professor E. Bujalance for his helpful comments and suggestions. The authors are partially supported by CICYTPB980017. 


\section{REFERENCES}

[1] N. L. Alling and N. Greanleaf, Foundations of the theory of Klein surfaces, Trans. Amer. Math. Soc. 283 (1984), 423-448.

[2] E. Bujalance and J. J. Etayo, A characterization of q-hyperelliptic compact planar Klein surfaces, Abh. Math. Sem. Univ. Hamburg 58 (1988), 95-102.

[3] E. Bujalance, J. J. Etayo, and J. M. Gamboa, Elliptic-hyperelliptic Klein surfaces, Mem. Real Acad. Ci. Exact. Fís. Natur. Madrid 19 (1985), 89 (Spanish).

[4] _ Hyperelliptic Klein surfaces, Quart. J. Math. Oxford Ser. (2) 36 (1985), no. 142, 141-157.

[5] E. Bujalance, J. J. Etayo, J. M. Gamboa, and G. Gromadzki, Automorphism Groups of Compact Bordered Klein Surfaces. A Combinatorial Approach, Lecture Notes in Mathematics, vol. 1439, Springer-Verlag, Berlin, 1990.

[6] J. A. Bujalance, Normal subgroup of even index in an NEC group, Arch. Math. (Basel) 49 (1987), no. 6, 470-478.

[7] _ Hyperelliptic compact non-orientable Klein surfaces without boundary, Kodai Math. J. 12 (1989), no. 1, 1-8.

[8] W. J. Harvey, On branch loci in Teichmüller space, Trans. Amer. Math. Soc. 153 (1971), 387-399.

[9] A. M. Macbeath, The classification of non-Euclidean plane crystallographic groups, Canad. J. Math. 19 (1967), 1192-1205.

[10] C. Maclachlan, Smooth coverings of hyperelliptic surfaces, Quart. J. Math. Oxford Ser. (2) 22 (1971), 117-123.

[11] D. Singerman, Automorphisms of compact non-orientable Riemann surfaces, Glasgow Math. J. 12 (1971), 50-59.

[12] _ On the structure of non-Euclidean crystallographic groups, Proc. Cambridge Philos. Soc. 76 (1974), 233-240.

[13] _ Symmetries of Riemann surfaces with large automorphism group, Math. Ann. 210 (1974), 17-32.

[14] H. C. Wilkie, On non-Euclidean crystallographic groups, Math. Z. 91 (1966), 87-102.

[15] H. Zieschang, E. Vogt, and H.-D. Coldewey, Surfaces and Planar Discontinuous Groups, Lecture Notes in Mathematics, vol. 835, Springer, Berlin, 1980.

J. A. Bujalance: Departamento de Matemáticas Fundamentales, Facultad de CienCias, UNED, PASEo Senda Del Rey 9, 28040 MAdRid, Spain

E-mail address: jbujalan@mat.uned.es

B. Estrada: Departamento de Matemáticas Fundamentales, Facultad de Ciencias, UNED, PASEo SENDA DEL Rey 9, 28040 MADRID, SPAIN

E-mail address: bestra@mat.uned.es 


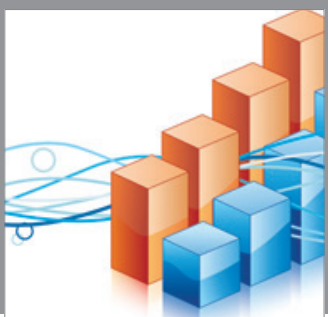

Advances in

Operations Research

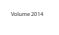

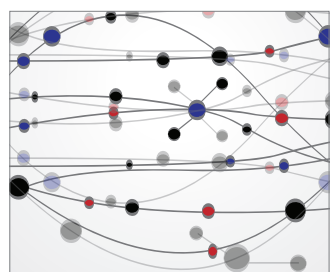

\section{The Scientific} World Journal
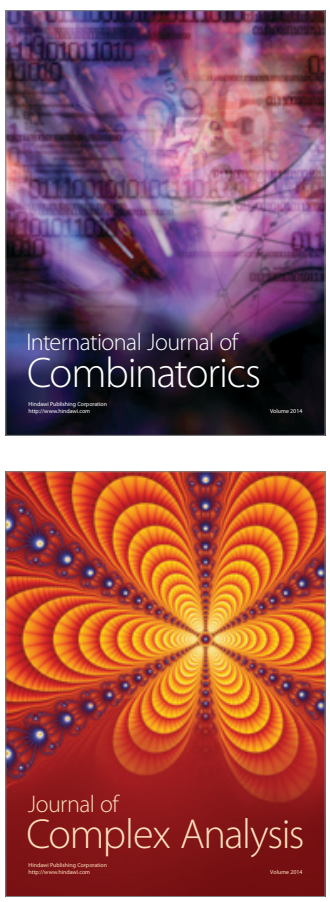

International Journal of

Mathematics and

Mathematical

Sciences
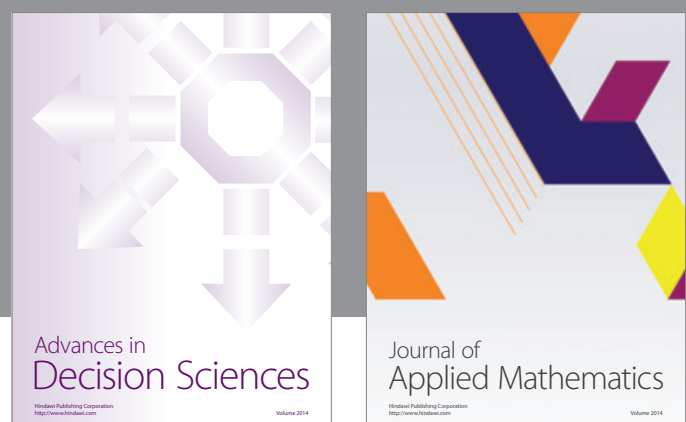

Journal of

Applied Mathematics
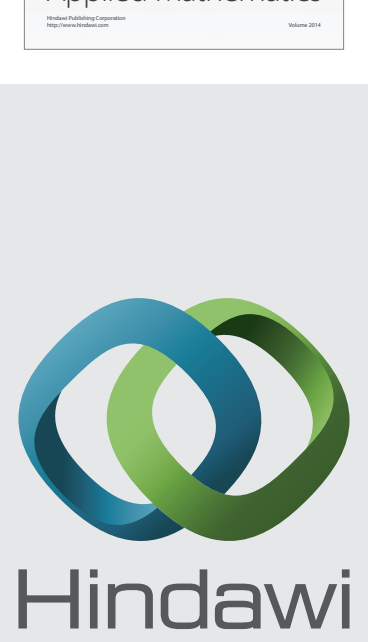

Submit your manuscripts at http://www.hindawi.com
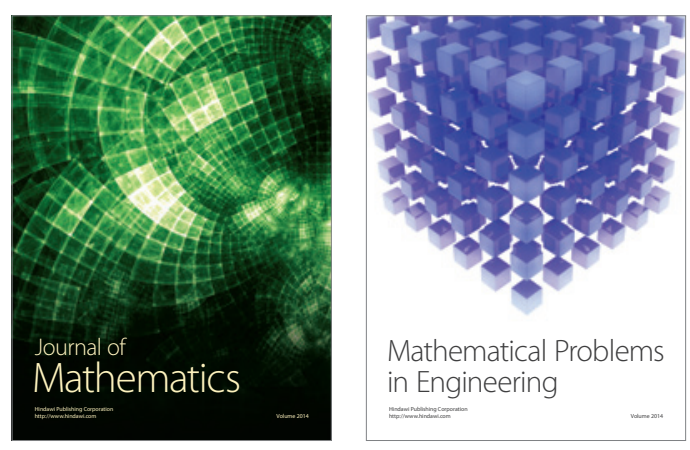

Mathematical Problems in Engineering
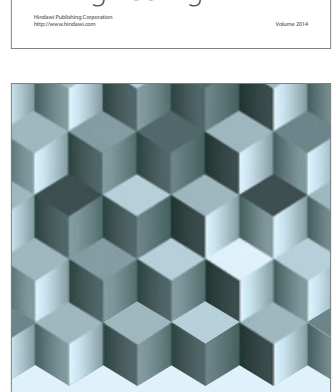

Journal of

Function Spaces
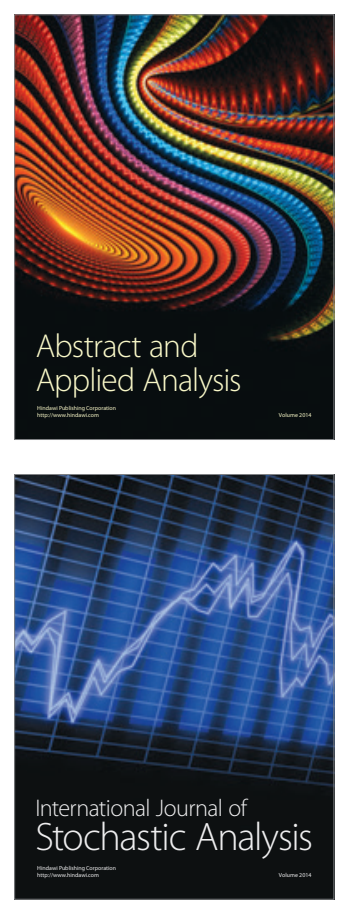

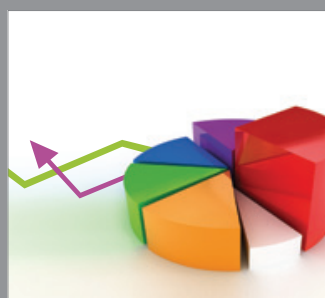

ournal of

Probability and Statistics

Promensencen
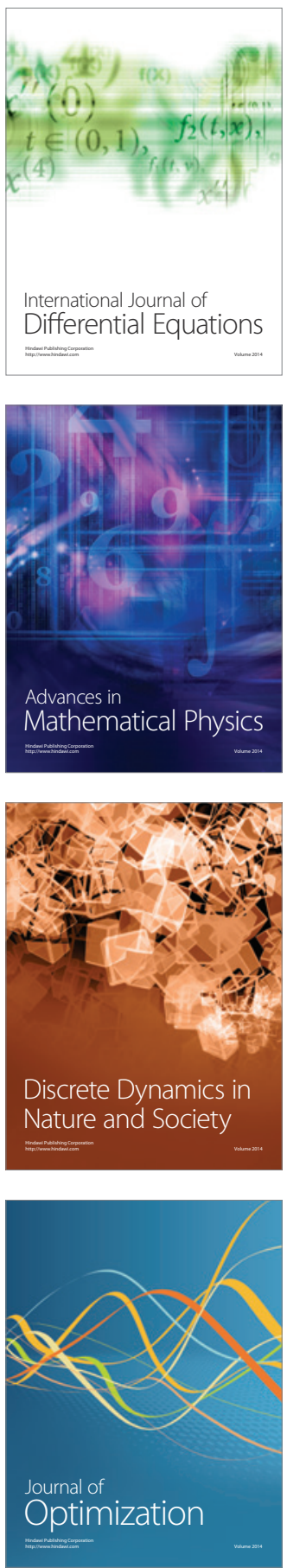